\title{
A Case of Thoracic Disc Herniation Effectively Treated with Interventional Radiology Computed Tomography- Guided Percutaneous Endoscopic Radiofrequency Annuloplasty Using a Disc-Fx®
}

\author{
Takayuki Kobayashi*, Hiroyuki Uchino, Kiyosige Ohseto, Yukihiko Ogihara, Hidekimi Fukui, \\ Shusuke Sekine, Yusuke Ishida and Aya Kawachi \\ Department of Anesthesiology, Tokyo Medical University Hospital, Japan
}

*Corresponding author: Takayuki Kobayashi, Department of Anesthesiology, Tokyo Medical University Hospital. 6-7-1 Nishisinjuku, Sinjuku-ku, Tokyo 160-0023, Japan

ARTICLE INFO
Received: 㓞 January 27, 2022
Published: 幽 February 04, 2022

Citation: Takayuki Kobayashi, Hiroyuki Uchino, Kiyosige Ohseto, Yukihiko Ogihara, Hidekimi Fukui, et al. A Case of Thoracic Disc Herniation Effectively Treated with Interventional Radiology Computed Tomography-Guided Percutaneous Endoscopic Radiofrequency Annuloplasty Using a Disc-Fx®. Biomed J Sci \& Tech Res 41(4)-2022. BJSTR. MS.ID.006636.

Keywords: Thoracic Disc Herniation; Percutaneous Discectomy; Interventional Radiology Computed Tomography

\begin{abstract}
Thoracic disc herniation is rare compared with cervical/lumbar disc herniation, accounting for only $0.25 \%$ to $1.00 \%$ of all patients with disc herniation. A patient consulted another hospital with lightning pain in the right flank, and a few days later was referred to our department. On physical examination, pressure pain was the most marked at the Th9 to 12 areas. Magnetic resonance imaging of the lumbar vertebrae led to a diagnosis of multiple disc herniation involving the Th9/10 to L4/5 areas. To treat the Th12/L1 herniated discs, intercostal nerve/epidural blocking was performed, but was ineffective. Therefore, blocking of the right Th12 nerve root was conducted under fluoroscopy. The symptoms slightly subsided. Subsequently, Computed Tomography - Discography was performed, leading to a definitive diagnosis of right lateral herniation at the Th12/L1 level. Percutaneous discectomy of the thoracic vertebrae may cause complications, such as pneumothorax and intervertebral arthrosis; therefore, operations must be carefully conducted. In the present case, we performed Interventional Radiology Computed Tomography-guided Percutaneous Endoscopic Radiofrequency Annuloplasty using a Disc-Fx®, although there are few reports on this treatment in Asia, but not so many worldwide yet. Subsequently, the patient's pain was reduced, with the Numerical Rating Scale (NRS) score of 1-2/10, and there were no complications, such as pneumothorax.
\end{abstract}

\section{Introduction}

In 1972, Hijikata et al. established percutaneous discectomy (PD), which is a procedure aiming at symptom relief for disc herniation through a reduction in nerve root irritation associated with a decrease in intervertebral disc pressure [1,2]. Furthermore, herniation reduction is achieved through secondary cicatricial contraction of the intervertebral discs. PD has subsequently been modified to be a safer, noninvasive procedure, and has been applied in various forms, such as with the use of laser, including the Disc-
$\mathrm{Fx}^{\circledR}$. The Disc-Fx ${ }^{\circledR}$ facilitates nucleus pulposus transpiration or fiber ring thermocoagulation in addition to conventional nucleus pulposus extenteration using forceps (Hijikata's procedure) [3-5]. In this study, we report a patient for whom Interventional RadiologyComputed Tomography (IVR-CT)-guided Percutaneous Endoscopic Radiofrequency Annuloplasty (PERA) was performed for the thoracic disc herniation to reduce the incidence of complications and improve treatment results, leading to a favorable outcome [6]. 


\section{Case Report}

The patient was a 34 -year-old man (height: $167 \mathrm{~cm}$; weight: $74.4 \mathrm{~kg}$ ), with a chief complaint of lightning pain in the right flank. He had no significant medical history. The patient consulted the Department of Anesthesia of another hospital with bilateral dorsal lower back pain, which had persisted for 1 year, and was treated with medication for 5 months. However, he stopped consulting the outpatient clinic due to symptom relief. 6 days before visiting our hospital, he again experienced lightning pain in the right flank appeared. 3 days before visiting our hospital, he consulted the Emergency Outpatient Unit of another hospital owing to exacerbation of the pain. Under a tentative diagnosis of ureteral calculi, an anti-inflammatory analgesic agent (diclofenac sodium suppository) was prescribed 2 days before visiting our hospital, he consulted the same hospital again, because there was no symptom relief. The presence of ureteral calculi was ruled out based on abdominal CT data. He was admitted with severe pain, and detailed examination and treatment were started. However, the presence of a disease in the field of internal medicine was ruled out. 1 day before visiting our hospital, he was discharged based on his wishes to consult another hospital. Although he consulted another local clinic, a definitive diagnosis could not be made. Next day, he consulted the outpatient clinic of our department. Physical examination on admission showed tenderness at the Th9 to 12 level.
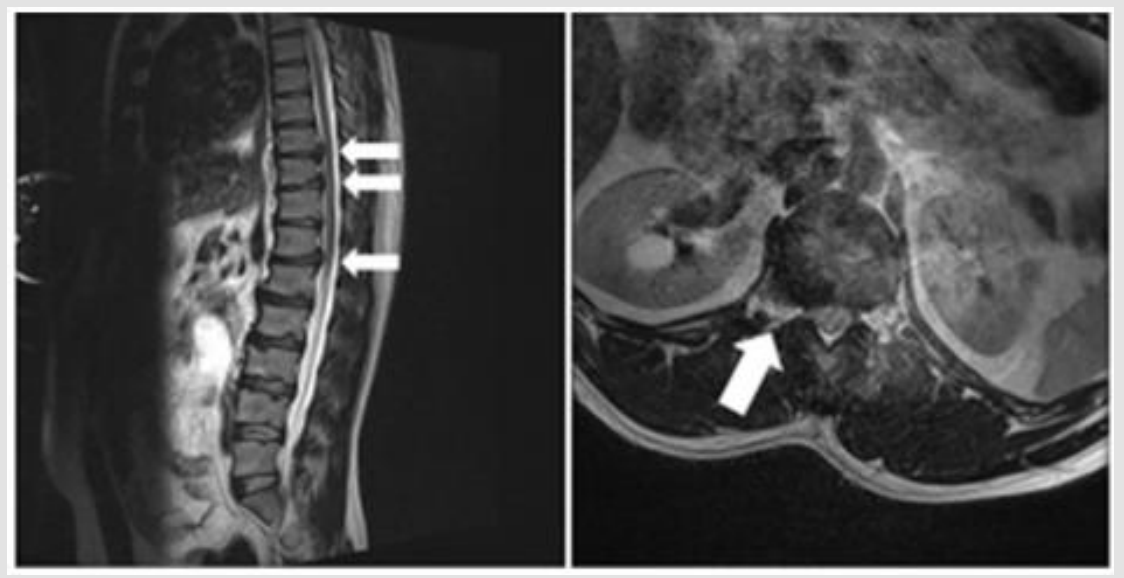

Figure 1:

a. MRI of the thoracic/lumbar vertebrae displayed multiple disc herniation involving the thoracic vertebrae to thoracolumbar junction (Th9/10, Th10/11, and Th12/L1). (arrows)

b. Coronal section of Th12/L1 showed a disc herniation (arrow).

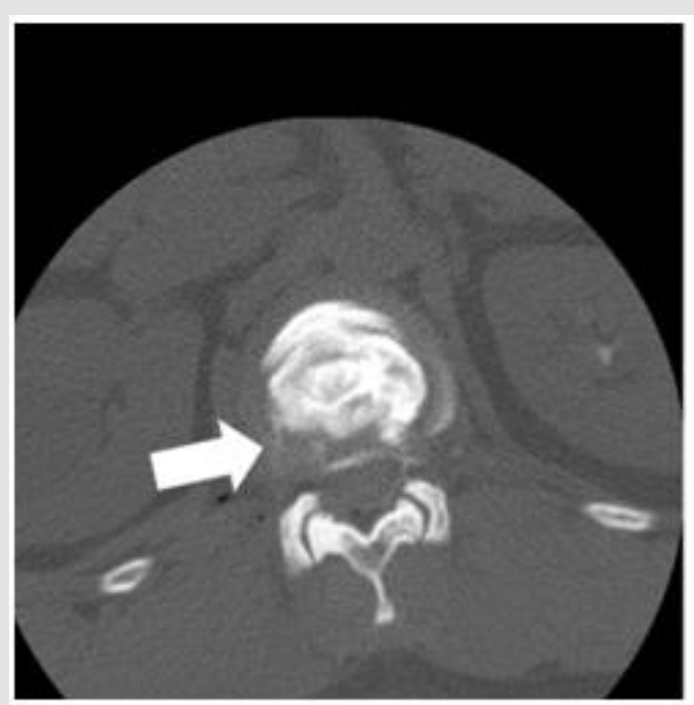

Figure 2: CT-discography led to a definitive diagnosis of lateral disc herniation at the Th12/L1 level (arrow). 
In particular, there was marked pain at the Th12 level, and allodynia was noted at the site of the pain. Hematological analyses did not demonstrate any abnormalities. The Numerical Rating Scale (NRS) score was 10/10, and the Self-rating Depression Scale (SDS) score was 41. The EuroQol-5 Dimension (EQ-5D) score was -0.594. X-ray of the thoracic/lumbar vertebrae displayed osteophyte formation, leading to a diagnosis of thoracic spondylosis deformans. Magnetic Resonance Imaging (MRI) of the thoracic/ lumbar vertebrae displayed multiple disc herniation involving the thoracic vertebrae to thoracolumbar junction (Th9/10, Th10/11, and Th12/L1), suggesting thoracic disc herniation as an etiological factor for lightning pain of the right flank (Figure 1). Outpatient treatment was started. Intercostal nerve (right Th10, 11, and 12)/epidural (Th12/L1) blockade was performed, but there was no improvement. Blockade of the right Th12 nerve root was conducted under fluoroscopy, but the pain remained, with an NRS score of 5/10. Subsequently, during CT-discography, the patient felt irradiating pain which was consistent with the pain he felt constantly. CT-discography led to a definitive diagnosis of lateral disc herniation at the Th12/L1 level (Figure 2). Therefore, IVR-CTguided PERA with a Disc-Fx ${ }^{\circledR}$ that features a patented navigational device with bipolar system which was specifically designed to access and treat the diseased portion of the disc was scheduled.

Under IVR-CT guidance, the point and route of insertion were determined, and a Disc-Fx ${ }^{\circledR}$ was inserted through an area $5 \mathrm{~cm}$ right to the spinous process at the Th12/L1 level to approach the intervertebral disc (Figure 3). Direct PD involving the herniated site was performed to treat the lateral herniation, and nucleus pulposus extenteration and thermocoagulation were conducted by DiscFx ${ }^{\circledR}$. There were no complications, such as pneumothorax thoracic spinal nerve root injury and haemorrhage. After treatment, the NRS score was reduced to $1-2 / 10$. Subsequently, follow-up was conducted by trigger point injection and drug therapy. 1 year after treatment, although there was a pain (NRS score was 2-3/10), there was no acute exacerbation of symptoms. The symptoms were calm, so it was decided to follow up at the clinic around the house, depending on the wishes of the patient.
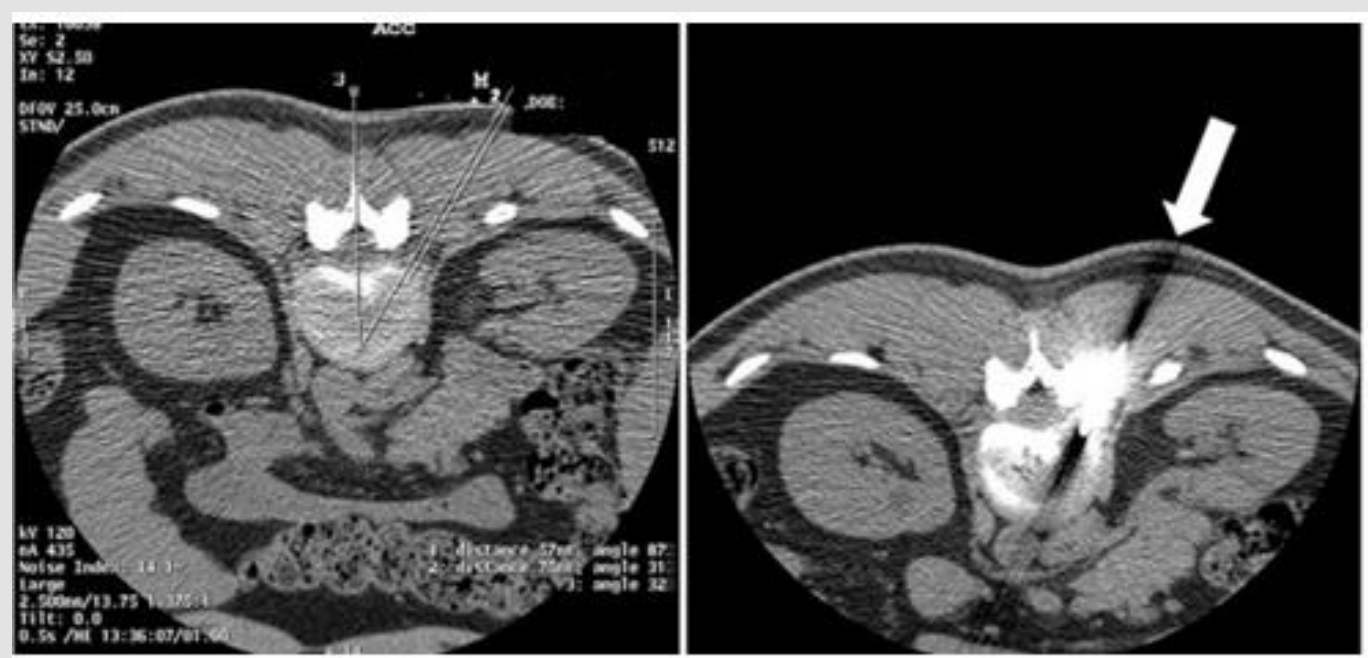

Figure 3:

a. Under IVR-CT guidance, the point and / route of insertion were determined.

b. A Disc-Fx® was inserted through an area $5 \mathrm{~cm}$ right to the spinous process at the Th12/L1 level to directly treat the lateral herniation (arrow).

\section{Discussion}

PD is an intermediate treatment procedure between surgical and conservative therapies, which was proposed by Hijikata in 1972 [1]. It reduces pain by decreasing the intervertebral disc pressure and reducing stimulation-associated nerve root pressure, degeneration of the disc, and cicatricial contraction of the disc. Advantages of PD include low-level invasiveness, a low incidence of postoperative complications, and shortening of the admission period. Treatment as an outpatient is possible. As complications, persistent inflammation of the nerve root and intervertebral arthrosis associated with a decrease in the intervertebral space have been reported. Several studies indicated the efficacy of PD combined with epidural/nerve root/intervertebral joint blocking for persistent inflammation of the nerve root [7]. Recently, PD has been improved to become safer and less invasive, and various techniques, including the use of laser, have been applied. As one such technique, the Disc-Fx ${ }^{\circledR}$ was developed. This technique uses Trigger-Flex ${ }^{\circledR}$ and Surgi-Max ${ }^{\circledR}$ for nucleus pulposus transpiration by percutaneous high-frequency intervertebral disc decompression or fiber ring thermocoagulation in the process of intra-disc highfrequency thermocoagulation in addition to conventional nucleus 
pulposus extenteration with forceps (Hijikata's procedure) $[3,4,8]$. In addition, this device facilitates the examination of the intra-disc nucleus pulposus and fiber ring under an endoscope.

Thoracic disc herniation is rare compared with cervical/lumbar disc herniation, accounting for $0.25 \%$ to $1.00 \%$ of all patients with disc herniation [8]. Symptoms of thoracic disc herniation vary, and include: dorsal chest pain, girdle pain, dysesthesia of the lower limbs (numbness, weakness), and myelopathy; therefore, an accurated diagnosis is difficult. In addition, treatment methods vary from conservative therapy to surgery. As this disease is rare, few studies have reported the results of treatment, and a consensus regarding treatment has not been reached. The present case was characterized by thoracic disc herniation and lateral herniation, which are rare. Therefore it was difficult to make a diagnosis. MRI of the thoracic/lumbar vertebrae, discography, and CT-discography led to a definitive diagnosis. The intervertebral foramens of the thoracic vertebrae are narrower than those of the lumbar vertebrae, and the lungs are present in the lateral regions; therefore, when performing PD for thoracic disc herniation, the risk of pneumothorax must be considered [9]. To reduce the risk of complications, we performed IVR-CT-guided PERA with a Disc-Fx ${ }^{\circledR}$, although there are still few reports on this procedure. The point and route of insertion were determined under IVR-CT guidance, facilitating individualdifference-matched fine adjustment. Effective treatment could be administered while reducing the risk of pneumothorax by directly puncturing and extirpating the site of lateral herniation.

In addition, spinal canal operations and intervertebral joint drilling was not required, in contrast with surgical procedures; therefore, it was possible to reduce the risk of complications, such as spinal canal adhesion and thoracic vertebral instability. 1 year after treatment, although there was a pain (NRS score was 2-3/10), because the disc herniation other than lateral disc herniation at the Th12/L1 level remained. However, of course there was no acute exacerbation of symptoms in TH12/L1 level. In conclusion, although the long-term efficacy should be further investigated, the present case suggests that IVR-CT-guided PERA with a Disc-Fx ${ }^{\circledR}$ is useful for treating patients with thoracic disc herniation.

\section{Conflicts of Interest}

The authors declare no conflicts of interest associated with this manuscript.

\section{References}

1. Kiyosige Ohseto, Masahiro Siotani, Yosikazu Naganuma, Hidetake Karasawa, Kenji Ohno, et al. (1996) Percutaneous Diskectomy for the Pain Treatment of Cervical and Lumbar Disk Hernias -Experience our Pain Clinic (in Japanese with English abstract). The Journal of the Japan Society of Pain Clinicians (JJSPC) 3: 1-6.

2. Wolfgang Bo rm, U Ba zner, R W Ko nig, T Kretschmer, G Antoniadis, et al. (2011) Surgical treatment of thoracic disc herniations via tailored posterior approaches. European Spine Journal 20(10): 1684-1690.

3. Chan Hong Park, Sang Ho Lee (2015) Efficacy of Nucleo-Annuloplasty Using Disc-FX® in Lumbar Disc Herniation. Journal of Spine 4(6).

4. Naresh Kumar, Aravind Kumar, Shah Siddharth M, Shah Sambhav P, Justin Tan, et al. Annulonucleoplasty using Disc- $\mathrm{FX}^{\circledR}$ in the management of lumbar disc pathology: Early results. International Journal of Spine Surgery 8: 18

5. Koichi Iwatsuki, Toshiki Yoshimine, Masao Umegaki, Kazuhiro Yoshimura, Masahiro Ishihara, et al. (2010) Treatment Status of Percutaneous Laser Disc Decompression for Lumbar Disc Herniation (in Japanese with English abstract). Japan Society for Laser Surgery and Medicine 31: 136-140.

6. Hiroaki Yamagami, Tetsushi Fukushima, Fukashi Yanaidani (2002) Clinical Evaluation of Percutaneous Lumbar Discectomy with Nerve Blocking Therapy ; A Report of 455 Cases (in Japanese with English abstract). Journal of Japan Society of Pain Clinicians (JJSPC) 9: 62-69.

7. Sukeaki Kabayama, Kazunori Yone, Masao Yamaguchi, Takashi Sakou (1998) Long-term Follow-up Study of Radiographic Evaluation after Percutaneous Discectomy for Lumbar Disc Herniation (in Japanese with English abstract). Orthopedics \& Traumatology 47: 1078-1080.

8. Stefan Hellinger (2014) Treatment of contained lumbar disc herniations using radiofrequency assisted micro-tubular decompression and nucleotomy: Four year prospective study results. International Journal of Spine Surgery 8: 24.

9. Scott MW Haufe, Anthony R Mork, Morgan Pyne, Ryan A Baker Percutaneous laser disc decompression for thoracic disc disease: Report of 10 cases. International Journal of Medical.
ISSN: 2574-1241

DOI: $10.26717 /$ BJSTR.2022.41.006636

Takayuki Kobayashi. Biomed J Sci \& Tech Res

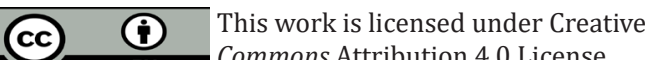

Submission Link: https://biomedres.us/submit-manuscript.php

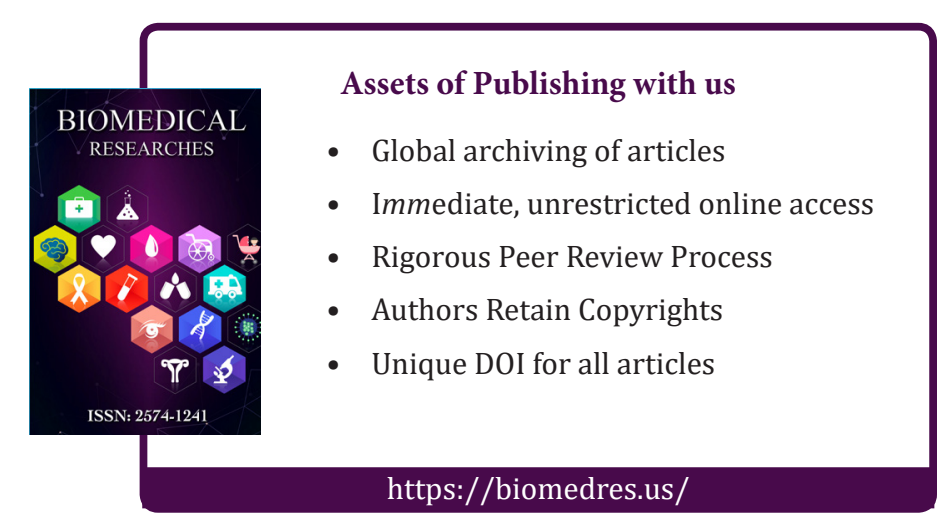

